\title{
Efficient Sensing Approaches for High-density Memristor Sensor Array
}

\author{
Adedotun Adeyemo, Jimson Mathew, Abusaleh Jabir, Corrado Di Natale, Eugenio Martinelli, Marco Ottavi
}

\begin{abstract}
Recent research shows ever growing inter-est in the potential applications of memristive devices. Among the many proposed fields, sensing is one of the most interesting as it could lead to unprecedented sen-sor density and ubiquity in electronic systems. In this paper, a framework for efficient gas detection using mem-ristor crossbar array is proposed and analysed. A novel Verilog-A based memristor model that emulates the gas sensing behaviour of doped metal oxides is devel-oped for simulation and integration with design au-tomation tools. Using this model, we propose and anal-yse three different gas detection structures based on ar-ray of memristor-based sensors. Gas presence together with some of its properties can be detected using re-sistance changes and spatial information from one or group of memristive sensors. Our simulation results show that depending on the organisation of the memristive elements and the sensing method, the response of the sensor varies providing a broader design space for future designers. For instance, with a $8 \times 8$ memristor sensor array, there is a ten times improvement in the accuracy of the sensor's response when compared with a single memristor sensor but at the expense of extra area overhead.
\end{abstract}

Keywords: memristor, gas sensor, memory, Verilog-A, crossbar array, metal oxide

\footnotetext{
A. Adeyemo, A. Jabir

Dept of Computing and Communication Technologies, Ox-ford Brookes University, Oxford, OX3 OBP UK

E-mail: 13121679@brookes.ac.uk

J. Mathew

Dept of Computer Sci. and Engr., Indian Institute of Tech-nology Patna, Patna 801103, India

M. Ottavi, C. Di Natale, E. Martinelli

Electronic Engineering, University of Rome "Tor Vergata" Rome 00133, Italy
}

\section{Introduction}

The gas sensing properties of metal oxide semiconduc-tors have been widely studied over the years [1-4]. With respect to other sensor technologies they are simple, inexpensive, miniaturisable and of good sensitivity [5]. Despite their simple operation, they are scarcely selec-tive and might be required to work at high tempera-ture [6]. However, advances have been made in some of these areas. Recently, authors in [7] demonstrated that nanomaterials metal oxide gas sensor can perform op-timally at room temperature [8]. Gas sensors are par-ticularly important in preventing the spread of gases that are harmful to organic life and also in the de-tection of oxygen deficiencies in environments where they are required. $\mathrm{T}_{\mathrm{i}}$ metal oxide semiconductor, which form the crux of this paper, was used by HP Labs to fabricate the first physical memristor device [9]. HP's memristor was made of a thin film $\mathrm{T} \mathrm{iO}_{2}$ sand-wiched between platinum electrodes. $\mathrm{T}^{\mathrm{iO}}{ }_{2}$ has specif-ically received extensive attention in various applica-tions such as photovoltaics [10], photocatalysis [11] and sensors [12]. In [13], relative humidity sensors were tested by employing vertically aligned $\mathrm{T}_{2}$ nanotubes array film produced using electro-chemical anodization of ti-tanium foil followed by a nitrogen-doping process. Mem-ristive biosensors are reported in [14], where memristive effects were registered on silicon nanowire. They also fabricated nanowires using lithographic technique that allows precise and selective etching at the nanoscale. The use of a single memristor cell as a sensor was demon-strated experimentally in [14] but single device sensors are usually susceptible to measurement errors due to issues such as a) instability of sensor response, b) ir-reproducibility of the sensor response among different devices and c) difficulty in maintaining high resolution 
response over a range of gas concentration. Cause measurement errors in sensors could be reduced $s$ tically by taking multiple measurements from the sensor or from different independent sensors [15]. paper explores memristor-based sensor structures crossbar architecture with emphasis on the memri: response to gas presence. A fundamental question tha work poses and attempts to answer is, given a fixed arr memristors, what is the best arrangement to achieve $b$ and reliable response. In particular, we investigate if better to have: 1) fewer sensor ele-ments with a accuracy; 2) large array of sensors with a high response and reliability; or 3) a trade off between the previous extremes. The critical ar-gument is that when the size number of sensors in the crossbar increases (decreases), the performance of the overall sensor structure improves (degrades) in similar fashion. In achieving this aim, a novel Verilog-A memristor sensor model is developed and three differ-ent crossbar sensing structures are analysed with this model.

The rest of the paper is organised as follows: Sec-tion 2 introduces the memristive device as well as mem-ristor's application in gas sensing and other preliminary concepts related to this work. Section 3 presents a de-scription of the proposed Verilog-A memristor model. In section 4, we show a more detailed analysis of the crossbar architecture and some key sensing problems are discussed. Section 5 describes the proposed struc-tures for gas sensing using memristor arrays. Further analysis of simulation results were presented in section 6 and section 7 concludes the paper.

\section{Background}

\subsection{Memristor}

The use of metal oxides as an insulating layer in met-al/ insulator/metal (MIM) devices have received signif-icant attention in resistive memory design because of their diverse electrical abilities. Memristor is a novel two-terminal passive electrical device (alongside the ex-isting trio of resistor, inductor and capacitor) whose ex-istence was originally proposed by Leon Chua in 1971 [16]. The first physical MIM memristor was fabricated by HP Labs, it consists of a thin film of semi-doped tita-nium dioxide $\left(\mathrm{T} \mathrm{iO}_{2}\right)$ sandwiched between two platinum electrodes. Memristor stores data as resistance and the resistance value of the device can be changed by apply-ing a voltage greater than its threshold voltage. This causes the device to switch between High Resistance State (HRS) $R_{\text {off }}$ and Low Resistance State (LRS) $\mathrm{R}_{\text {on }}$ depending on the amplitude, polarity and duration of

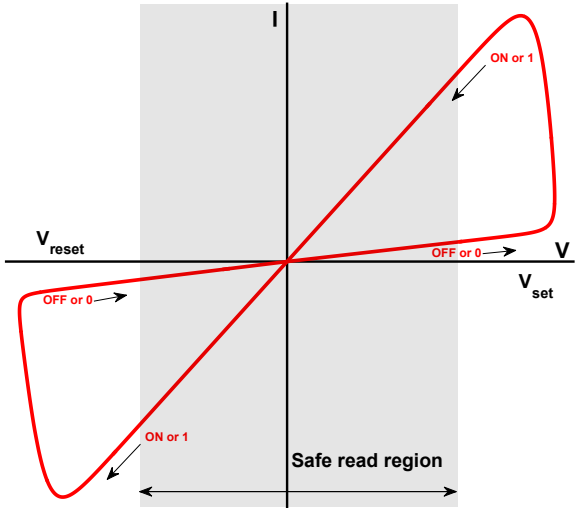

(a)

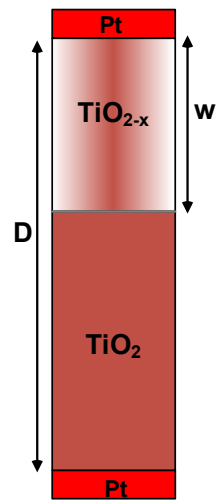

(b)
Fig. 1: (a) Memristor linear I-V characteristics showing transitioning between high and low resistance state in the presence of sinusoidal voltage source. (b) Structure of the $\mathrm{TiO}_{2}$ based memristor fabricated by HP Labs.

the voltage applied. The switching from $R_{\text {on }}$ to $R_{\text {off }}$ is regarded as the RESETing process whereas the transi-tion from $R_{\text {off }}$ to $R_{\text {on }}$ is known as the SETing process (the write voltage must be greater or equal to the SET (RESET) threshold voltage) as shown in Fig. 1a.

\subsection{Memristor as Gas Sensor}

The use of metal oxide nanoscale devices as gas sensors and biosensors has grown in recent years [13]. Memris-tor is among the nano-devices that have recently been investigated for their use as a sensing element because it primarily consists of metal oxide [14]. Apart from $\mathrm{T} \mathrm{iO}_{2}$, memristive devices can be fabricated using dif-ferent metal oxide semiconductors such as aluminium oxide $\left(\mathrm{Al}_{2} \mathrm{O}_{3}\right)$ [23], copper oxide $\left(\mathrm{Cu}_{2} \mathrm{O}\right)$ [24], silicon oxide (SiO) $[25,50]$ among many others [26-28]. A typ-ical structure of $\mathrm{T} \mathrm{iO}_{2}$ based memristor is the HP Labs memristor shown in Fig. $1 b$ and described in $[29,30]$. Memristor's resistance variation can be captured to in-dicate presence of gases and it is the building block of recently shown memristor sensors. Authors in [31] fabri-cated and demonstrated that a $\mathrm{Pt} / \mathrm{T} \mathrm{iO}_{2} / \mathrm{P}$ t memristor can effectively be used as an hydrogen sensor at room temperature. When a certain concentration of gas is directed towards the surface of a memristor, its resis-tance might be altered depending on the semiconduct-ing material, causing a momentary change in output of the associated read circuitry. Modelling this resistance changing behaviour in a sensor array is the basis of a smart sensing system. By building different structural models and then modelling the read behaviour and re-sponse of the structure, it is possible to evaluate the 


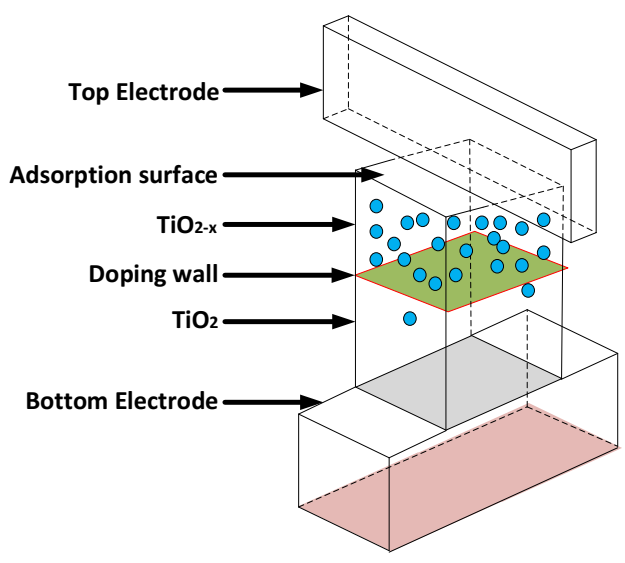

Fig. 2: Structure of memristor for sensing applications. performance of a smart sensing system. A structural

Table 1: Effect of increasing gas concentration on the resistance of semiconductor metal oxide [32].

\begin{tabular}{|l|c|c|c|}
\hline \multirow{2}{*}{\multicolumn{2}{|c|}{ Classification }} & \multicolumn{2}{c|}{ Gas Type } \\
\cline { 3 - 4 } & Reducing & Oxidising \\
\hline \multirow{2}{*}{ Semiconductor } & p-type & $\uparrow$ & $\downarrow$ \\
\cline { 2 - 4 } & n-type & $\downarrow$ & $\uparrow$ \\
\hline
\end{tabular}

analysis such as the ones presented in this paper could be used in the early stages of new smart sensing system development, to simulate the overall behaviour and in identifying any vulnerabilities before any actual system is developed. If problems are identified sooner during development, then less time and cost will be required to fix those problems. The basic mode of operation of a metal oxide gas sensor is the control of the surface potential barrier by the adsorbed surface-charge. The interaction of a target gas with the surface of a metal oxide sensor results in a change in the concentration of charge carriers in the material. The change in the concentration causes resistivity alteration in the metal oxide. In order for a memristor to be effective as a gas sensor, a slight modification will be necessary into de-vices fabricated for sensing purpose.

The physical memristor will feature a partially cov-ered top terminal as shown in Fig. 2 to allow gas inter-action with the semiconductor layer. The degree and direction of resistance change depends on the semicon-ductor material in the sensor as well as the subject gas. Table 1 shows a summary of how the resistance of metal oxides react to the presence of different gases. This pa-per focuses on $T$ $\mathrm{iO}_{2}$ based memristor. $\mathrm{T} \mathrm{iO}_{2}$ is regarded as a reliable chemical sensor because of its better re-sponse rate, low cost, fast response time and stabil-ity [7, 33, 34]. Gas adsorbed by the surface of $\mathrm{a} \mathrm{T}_{\mathrm{iO}}$

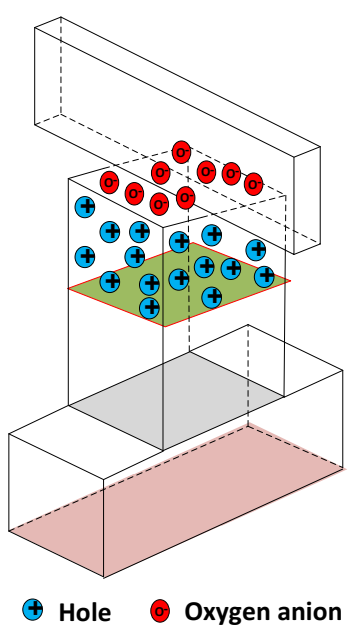

(a)

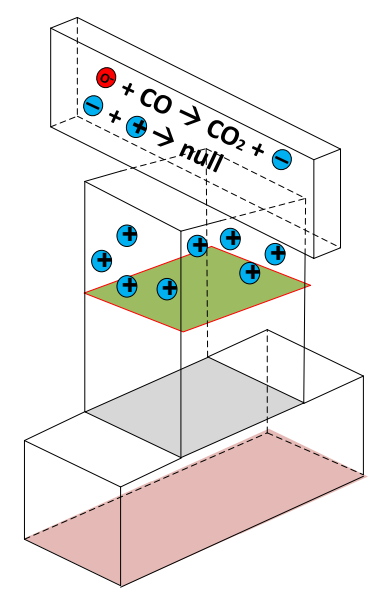

$\Theta$ Electron

(b)
Fig. 3: (a) Oxygen anion are adsorbed to the surface of the memristor on exposure to air (b) In the presence of a reducing gas such as $\mathrm{CO}$, the oxygen anion react with the gas to release electron that are injected into the thin film.

film increases or decreases the resistance of the film as a result of interactions among electrons in the film. We propose to use this memristor as a sensor by initialis-ing the memristor to the $\mathrm{R}_{\text {on }}$ state by applying a high positive voltage to the positively charged ${\mathrm{T} \mathrm{iO}_{2-x}}$ layer. This repels the oxygen vacancies in the $\mathrm{TiO}_{2-x}$ region further into the $\mathrm{T}$ $\mathrm{iO}_{2}$ layer, thereby making the de-vice almost completely filled with the more conductive $\mathrm{T}_{\mathrm{iO}} \mathrm{O}_{2-\mathrm{x}}$ from the surface. The total resistance (memris-tance) of the memristor is determined by two variable resistors $R_{\text {off }}$ and $R_{\text {on }}$ representing the resistance of the undoped $\left(\mathrm{T} \mathrm{iO}_{2}\right)$ and the doped $\left(\mathrm{T} \mathrm{iO}_{2-\mathrm{x}}\right)$ regions (Fig. $\left.1 \mathrm{~b}\right)$ of the memristor respectively. $\mathrm{T} \mathrm{iO}_{2-x}$ is a p-type semiconductor and its resistivity $\left(R_{\text {on }}\right)$ decreases (increases) in the presence of oxidising (reducing) gases. The concentration of charge carrier in the $\mathrm{T} \mathrm{iO}_{2}$ thin film will increase (decrease) in the presence of an oxidis-ing (reducing) gas since holes (electrons) are generated from the interaction of such gas with the oxygen anion $\left(\mathrm{O}^{-}\right)$adsorbed at the surface of the device [3, 34, 35]. Fig. 3a shows oxygen ion adsorbed to the surface of the memristor on exposure to air. Whenever a reduc-ing gas such as carbon monoxide (CO) is exposed to the surface of the memristor sensor, oxidation reaction takes place between the $\mathrm{CO}$ and the oxygen anions to emit electrons as shown in Fig. 3b. The emitted elec-trons in turn nullifies the positively charged carrier in the $\mathrm{T}^{\mathrm{iO}} \mathrm{O}_{2-\mathrm{x}}$ region thereby increasing its resistance and the resistance of the entire device. 


\section{Verilog-A Model for Memristor Sensor}

Prior to analysis of memristor-based structures suitable for gas sensing, there is the need to develop an effective memristor sensor model as there is not a known one in existence. This model accounts for gas concentration as an input to the memristor model as this form the basis for memristor's application in gas detection. Generally, when semiconducting metal oxide (such as $\mathrm{T} \mathrm{iO}_{2}$ based memristor) are used as gas sensor, the main cause of change of the sensor's resistance is as a result of the loss (gain) of free charge carriers (electrons or holes) from (to) the semiconductor to (from) its surface [36]. Any of the memristor models based on a gas sensitive

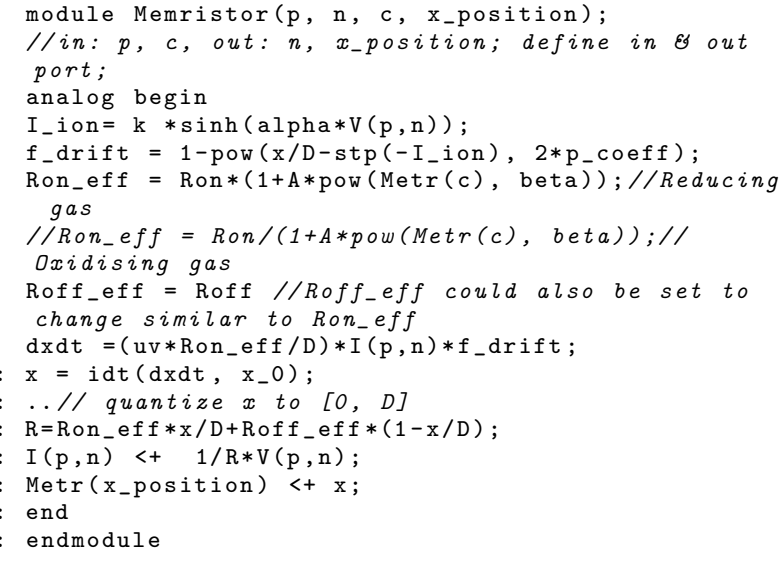

Fig. 4: Verilog-A description for proposed memristor model for sensing application.

metal oxide semiconductor can be adapted for the purpose of simulating gas sensor. We present an adaption of the physical memristor fabricated by HP labs for use as a memristive gas sensor. As earlier mentioned, HP's memristor consist of two regions; the perfect and less conductive $\mathrm{TiO}_{2}$ and the conductive $\mathrm{T} \mathrm{iO}_{2-x}$ with oxy-gen vacancies (holes) as shown in Fig. 1b. While the practical viability of the $\mathrm{T} \mathrm{iO}_{2}$ model has been contested in memory design, it is sufficient for sensor simulations as the resistance changes de-pend primarily on the gas concentration. Also, the $\mathrm{T}_{\mathrm{iO}}$ model has been fabricated and exper-imentally demonstrated to be effective for gas detection [31]. The positively charged oxygen vacan-cies makes the $\mathrm{T} \mathrm{iO}_{2-x}$ material conductive [9]. Gas in-teraction will occur at the surface of the $\mathrm{T}_{\mathrm{iO}_{2-x}}$ layer where the number of positively charged holes will in-crease or decrease depending on the properties of the subject gas (Table 1). We assume the ion mobility rate will remain at its average value.

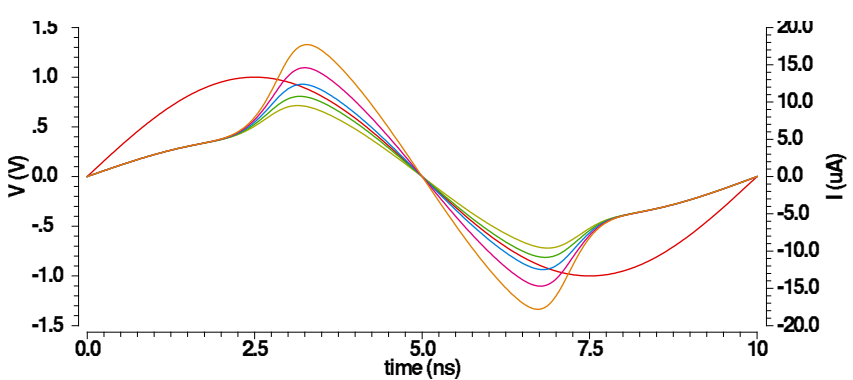

(a)

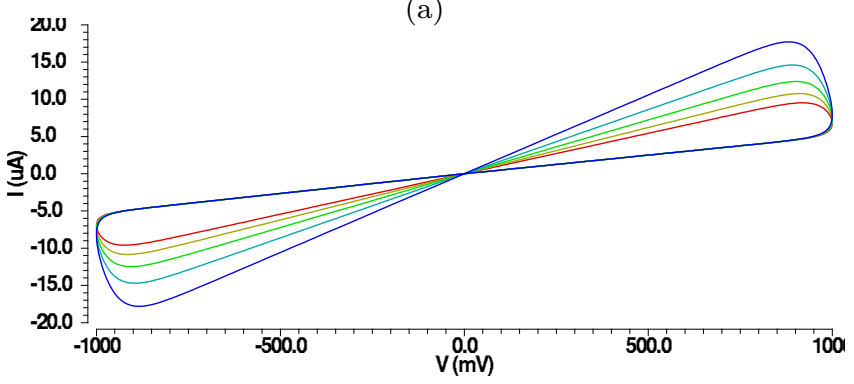

(b)

Fig. 5: (a) The applied voltage (red) and resulting cur-rents (other colours) as a function of time and gas concentration for the memristor model (b) I-V characteris-tics of proposed memristor model over 5 steps of an ox-idising gas concentration ( 0 ppm (red curve) - 1000 ppm (blue curve)). An adaptation of Eqn. 1 was used, ini-tial $R_{\text {on }}$ and $R_{\text {off }}$ are $100 \Omega$ and $200 \mathrm{~K} \Omega$ respectively, $\beta=1$ [37] and $A=$ $4.2 \times 10^{-4}$.

The Verilog-A model listed in Fig. 4 will facilitate easy integration and simulation of memristor-based sen-sors with design automation tools. For brevity, only the main functions are shown. The model was built on the basic memristor model presented in [38] and further ex-panded in [39]. The Biolek window function [40] to en-sure state variable $x$ remains within the 0 and $D$ bound-ary is shown in line 5 . The Butler-Volmer equation in line 4 is used to introduce some degree of non-linearity to the currentvoltage (I-V) characteristics with pro-grammable thresholds. $k$ and $\alpha$ are fitting constants for characterising the memristor's state. The response (S) of sensors to gas presence is often defined in vari-ous forms such as $S=R_{\text {init }} /$ $R_{\text {final, }}, S=R_{\text {final }} / R_{\text {init }}, S=\left|R_{\text {init }}-R_{\text {final }}\right| / R_{\text {init }}, S=\left|R_{\text {init }}-R_{\text {final }}\right| /$ $R_{\text {final, }}$, where $R_{\text {final }}$ and $R_{\text {init }}$ are the final and initial resistance of the sensor after and before exposure to gas respectively. The value of $R_{\text {on }}$ changes to $R_{\text {on eff }}$ using equation in line 6 from the adaptable response model equation in Eqn. 1 [32, 37, 41] depending on the type of gas interacting with the memristor. Therefore, for any oxidising gas of concentration $\mathrm{C}$, the response of a gas sensor made of p-type semiconductor can be rep-resented directly by Eqn. 1. Clearly, the response of a p-type material to a reducing gas can also be repre- 


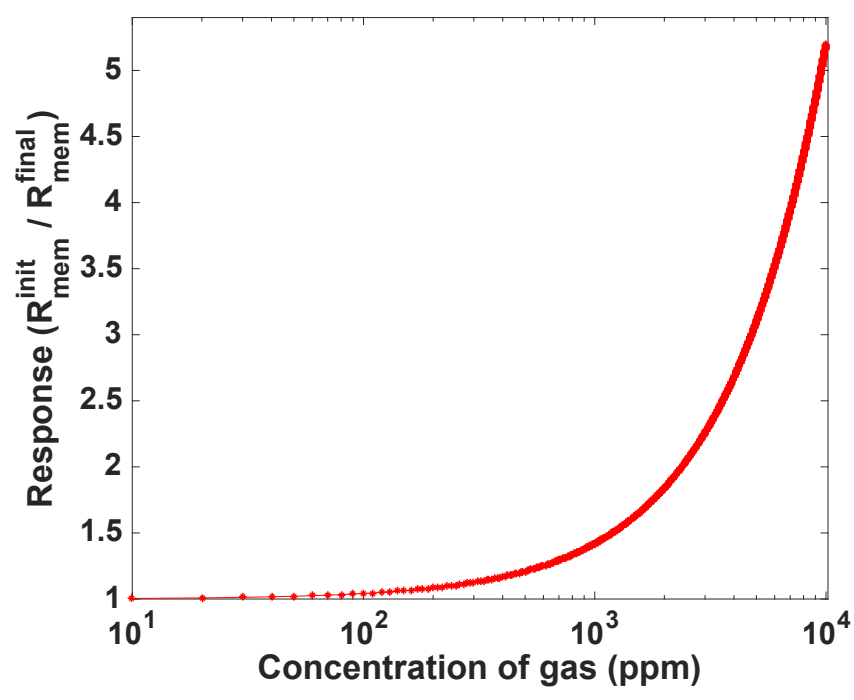

Fig. 6: Sensor response to different concentrations of an oxidising gas. $\mathrm{R}_{\mathrm{mem}}^{\text {init }}$ is calculated using Eqn. 2 with the initial values of $R_{\text {on }} R_{\text {off }}$. $R_{\text {mem }}^{\text {inal }}$ (Eqn. 3 ) is the memristance of the sensor after gas interaction, where $R_{\text {on }}$ changes to $R_{\text {on }_{e} f f}$.

sented by the inverse of Eqn. 1 .

$S=\frac{R_{\text {init }}}{R_{\text {final }}}=1+A[C]^{\beta}$

The response equation above is based on the concentration $\mathrm{C}$ (one of the three input ports in the model) of the target gas, Here, $A$ is the constant parameter or sensitivity coefficient for the material of the semicon-ductor and $\beta$ is the response order for the subject gas. $R_{\text {on }}\left(R_{\text {off }}\right)$ and $R_{\text {on eff }}$ ( $R_{\text {off eff }}$ ) in the Verilog-A model replace $R_{\text {init }}$ and $R_{\text {final }}$ respectively in Eqn. 1. Depending on the type of gas, Eqn. 1 can be adapted for either an oxidising or reducing gas as summarised in Table $1 . \mathrm{T} \mathrm{iO}_{2}$ is a n-type semiconductor metal oxide but can be transformed into a p-type by doping [34]. HP Lab's memristor can be regarded as a ptype metal oxide semiconductor material when used a gas sensor with the doped $\mathrm{T}_{\mathrm{iO}_{2-x}}$ region exposed to the subject gas.

The Verilog-A model is developed based on the generally acceptable assumption that only the region of the memristor directly exposed to the target gas experience a change in resistance [35,37]. The model thus uses the new $R_{\text {on eff }}$ and $R_{\text {off eff }}$ to compute the final memris-tance $\left(R_{\text {ment }}^{\text {fnal }}\right)$ of the device after exposure to the target gas using Eqn. 3 . The initial memristance of the device is calculated with $R_{\text {on }}$ as in Eqn. 2.

$$
\begin{aligned}
& R_{m e m}^{\text {init }}=R_{o n} \frac{w(t)}{D}+R_{o f f}\left(1-\frac{w(t)}{D}\right) \\
& R_{\text {mem }}^{\text {final }}=R_{\text {on_eff }} \frac{w(t)}{D}+R_{o f f \_e f f}\left(1-\frac{w(t)}{D}\right)
\end{aligned}
$$

where $0 \leq \mathrm{w}(\mathrm{t}) \leq \mathrm{D}$ is the time dependent state vari-able acting as a boundary between the doped and un-doped regions. $R_{\text {off }}$ eff and $R_{\text {on eff }}$ are the effective new resistance values of the two regions after exposure to gas. The memristor is initialised to $R_{\text {on }}(w=D)$ such that it constitutes mostly oxygen deficient $T$ $\mathrm{iO}_{2-\mathrm{x}}$. In-teraction of gases with the doped region changes $\mathrm{R}_{\mathrm{on}}$ to $R_{\text {on eff }}$ without shifting the state variable. The ex-tent of change to the resistance of the doped region depends on the carrier concentration and type of gas. As portrayed in the Verilog-A model, $R_{\text {off eff }} \approx R_{\text {off }}$ because the gas has negligible interaction with the un-doped region of the memristor, the Verilog-A model could also be adjusted to accommodate changes in $R_{\text {off }}$. The I-V characteristics of the developed model was plotted over a range of gas concentration to ensure the device still retains its memristive behaviour. A sinu-soidal voltage was applied to the device and the corre-sponding current responses shown in Fig. 5a are very similar to those reported in various experimental re-sults in literature $[31,40,42]$. The current-voltage (I-V) curves from the model indicating hysteresis is shown in Fig. 5b. The I-V curves shows results over a parametric range (0 ppm - $1000 \mathrm{ppm})$ of an oxidising gas concen-tration. From the I-V characteristics, the $R_{\text {off }}$ value remains almost constant because the gas interact less with the undoped layer of the memristor as previously explained. On the other hand, $R_{\text {on }}$ reduces to $R_{\text {on eff }}$ causing increased current as gas concentration increases as seen by the multiple coloured lines on the vertical plane of curve in Fig. 5b. The I-V characteristics were obtained through simulation in Cadence Virtuoso with a sinusoidal input voltage to the sensor element.

The authors in [49] presented the effects of temperature on the I-V characteristics of Tantalum-oxide-based resistive memories. Their results show

a $60^{\circ} \mathrm{C}$ temperature change triggering a negli-gible change in the hysteresis curve of the de-vice. The technique of [31] also showed that the $\mathrm{TiO}_{2}$ gas sensor can effectively respond to gas presence in the range $24-100^{\circ} \mathrm{C}$. In addition, it has been demonstrated in [48] that a current change caused by parasitic effects only move the I-V curve's pinch-point marginally. As the over-all current in the sensor is expected to be low, our model did not consider the effects of tem-perature changes. With the Verilog-A model firmly established, the sensing capability of a single isolated memristor was verified with an oxidising gas. Fig. 6 shows a single memristor's response as a function of gas concentrations ranging from $0 \mathrm{ppm}$ to $10^{4} \mathrm{ppm}$. Ex-pectedly, the response rate increases with increasing gas concentrations. 


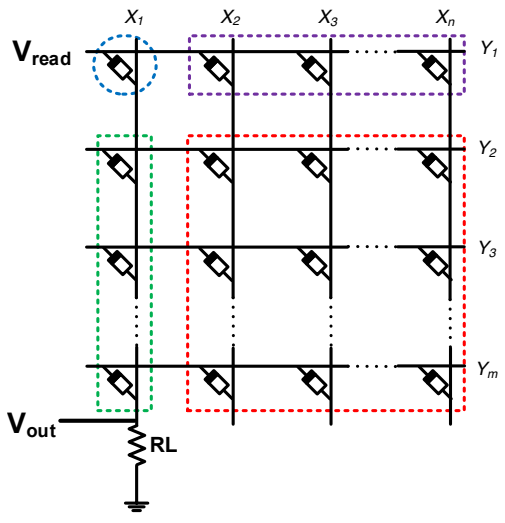

(a)

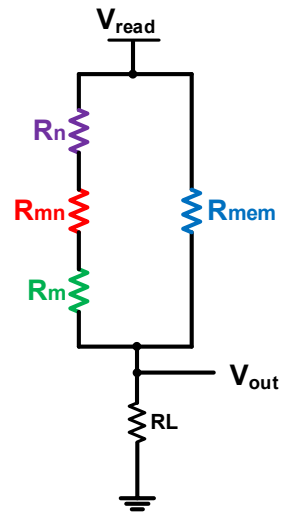

(b)
Fig. 7: (a) A typical crossbar architecture conditioned for reading. The cell circled in blue $\left(R_{\text {mem }}\right)$ is the target cell (b) Equivalent circuit of the ideal crossbar read operation showing the undesired parallel combination of neighbouring memristors with the target memristor to influence the read output.

For the development of the Verilog-A sensor model, we have made the following assumptions:

1. The memristor operates at a temperature sufficient for the resistance of the thin film to change in the presence of gases. Metal oxide sensors are able to operate at room temperature $[7,8,31]$.

2. The gas concentration causes an absolute change in the value of $R_{\text {on }}$ alone which causes no significant change to the position of the state variable $x$.

3. The resistance value of the memristor could be readout with or without the presence of the target gas using appropriate read circuitry.

4. Recovery time of memristor depends on the properties of the material in use.

\section{Gas Sensing with Crossbar Array}

As earlier mentioned, the use of a single memristor cell as a sensor has been demonstrated experimentally in previous publications [14, 21]. Measurement errors in sensors could be reduced statistically by taking mul-tiple measurements from the same sensor or from dif-ferent independent sensors [15]. Array of sensors also open up the possibility of detecting multiple gases in real time. The use of MOSFET based sensors in an ar-ray to improve performance was demonstrated in [43], where pairs of Pdand Pt-gate MOSFET were used to detect and analyse gas mixtures. Similarly, the use of commercially available $\mathrm{SnO}_{2}$ sensors in an array to detect and differentiate organic compounds was also ex- plored in [44]. These array of sensors make it possible to detect, quantify and differentiate multiple gases in real time. Fig. 7a shows a crossbar array with the cell circled in blue for sensing. The crossbar consists of a set of parallel nanowires perpendicularly placed on another set of parallel nanowires with a memristor cell inserted at every intersecting point of the wires. Each set of parallel wires represents the bitlines and wordlines. In order to sense the resistance of a memristor in a cross-bar memory array, a read voltage $V_{\text {read }}$ (less than the threshold voltage to switch the memristor) is applied to either the wordline or bitline and the other line is grounded through a load resistor (RL). The other lines can be left floating. However, this simple sensing tech-nique introduces the sneak-path problem that hinders independent sensing of each device in the array. The sneak-path problem is explained in the next section.

\subsection{Effect of Sneak-path on Crossbar Array Sensing}

Memristor crossbar architecture suffers from sneak-path because of the bidirectional flow of current in oxide based memristors. Sneak-paths are undesired current paths within the crossbar architecture that may lead to erroneous sensing of the resistance of a memristor cell in a crossbar array. Resistance of unselected cells combine to form a parallel resistance path with the resistance of the desired cell(s) as shown in the equivalent circuit in Fig. 7a [45]. The resistance model of any $m \times n$ mem-ristor crossbar array with one cell selected for sensing can be described as follows: Let $\mathrm{R}$ be the resistance of each cell in the array of Fig. 7a. Assuming the selected cell (blue) has a resistance of $R=R_{\text {mem }}$, the resistance of the three other groups of cells in the same schematic can be represented by $R_{m}$ (green), $R_{n}$ (purple) and $R_{m n}$ (red) as defined by Eqn. 4 - 6 respectively.

$$
\begin{aligned}
R_{m} & =\frac{\prod_{i=1, i \neq i_{s}}^{m} R_{i, j_{s}}}{\sum_{i=1, i \neq i_{s}}^{m} R_{i, j_{s}}} \\
= & \begin{cases}\frac{R}{m-1} & \text { if all } R_{i, j_{s}} \\
\frac{R_{o n} R_{o f f}}{(m-1) R_{o f f}-K_{o f f}\left(R_{o f f}-R_{o n}\right)} & \text { otherwise }\end{cases}
\end{aligned}
$$




$$
\begin{aligned}
& \prod^{n} \prod^{m} R_{i, j} \\
& R_{m n}=\frac{\sum_{j=1, j \neq j_{s}} \sum_{i=1, i \neq i_{s}}^{n} R_{i, j}}{\sum_{i=1, i}} \\
& =\left\{\begin{array}{l}
\frac{R}{(m-1)(n-1)} \\
\frac{R_{o n} R_{o f f}}{(m-1)(n-1) R_{o f f}-K_{o f f}\left(R_{o f f}-R_{o n}\right)}
\end{array}\right. \\
& R_{n}=\frac{\prod_{j=1, j \neq j_{s}}^{n} R_{i_{s}, j}}{\sum_{j=1, j \neq j_{s}}^{n} R_{i_{s}, j}} \\
& =\left\{\begin{array}{l}
\frac{R}{n-1} \\
\frac{R_{o n} R_{o f f}}{(n-1) R_{o f f}-K_{o f f}\left(R_{o f f}-R_{o n}\right)}
\end{array}\right.
\end{aligned}
$$

if all $R_{i_{s}, j}$ are equal otherwise

Here, $\mathrm{i}$ and $\mathrm{j}$ are the wordline and bitline index respectively; $i_{s}$ and $j_{s}$ are the selected wordline and bit-line respectively; $K_{\text {off }}$ is the number of memristors in the off state (logic 0 ) assuming $R_{i, j}$ is either $R_{\text {off }}$ or $R_{\text {on }}$ as it is the case in memristor memory arrays. In order to sense a device from the array, the selected row and column are connected to the read voltage $\left(\mathrm{V}_{\text {read }}\right)$ and ground respectively and the other lines are left floating. The sensing mechanism in Fig. 7a is the volt-age divider formed by the selected cell and the con-nected load resistance $\left(R_{L}\right)$. Ideally, the output voltage should depend largely on the content of the selected cell, $R_{\text {mem }}$. However, in the real case, sneak-path re-sistance from other unselected cells interfere with the output voltage. The total sneak path resistance $R_{\text {sneak }}$ in the array is a series combination of $R_{m}$, $R_{n}$ and $R_{m n}$ according to Eqn. 7 .

$R_{\text {sneak }}=R_{m}+R_{n}+R_{m n}$

The sneak path resistance $R_{\text {sneak }}$ combines parallelly with that of the selected cell $R_{\text {mem }}\left(R_{\text {off }}\right.$ or $\left.R_{o n}\right)$ to result in the effective resistance that forms a voltage divider with the load resistance $R_{L}$.

$R_{\text {mem eff }}=R_{\text {mem }}|| R_{\text {sneak }}$

The sensing margin or response $(\Delta V)$ parameter represents the figure of merit that makes it possible to differentiate between the two possible logic states $0\left(R_{\text {off }}\right)$ and 1 $\left(R_{o n}\right)$ of a memristor cell. The sensing margin is calculated as the difference between the output voltage of the $R_{o n}$ and $R_{\text {off }}$ states of the memristor as shown below:

$\Delta V=V_{\text {out }}^{1}-V_{\text {out }}^{0}$

Ideally, $\mathrm{V}_{\text {out }}$ should be computed according to Eqn. 10 where the output is strictly dominated by the selected memristor and the known load resistor and not Eqn. 11 but for sneak-path.

$V_{\text {out }}=V_{\text {read }} \times \frac{R_{L}}{R_{L}+R_{\text {mem }}}$

$V_{\text {out }}=V_{\text {read }} \times \frac{R_{L}}{R_{L}+R_{\text {mem_eff }}}$

Sneak-path effect makes it difficult to use each of the memristor in the crossbar array as individual sen-sor. Apart from degrading the sensing margin, existence of sneak paths also leads to increased power consump-tion. Sneakpaths also limit the maximum array size because sensing margin degrades severely as the array size increases as shown in Fig. 8. The figure shows how sneak path affects the sensing margin. For square arrays $(m=n)$, the margin deteriorates rapidly as the size in-creases. Hence the need to device techniques to avoid or leverage sneak-path effect in the design of memristor-based sensor arrays.

\section{Proposed Sensing Structures}

\subsection{The $m \times n$ Array Structure}

Sensing each memristor from the basic crossbar structure without an isolating device or extra line biasing is usually plagued by sneak-path as described in Sec-tion 4.1. In the presence of sneak-path, each memristor

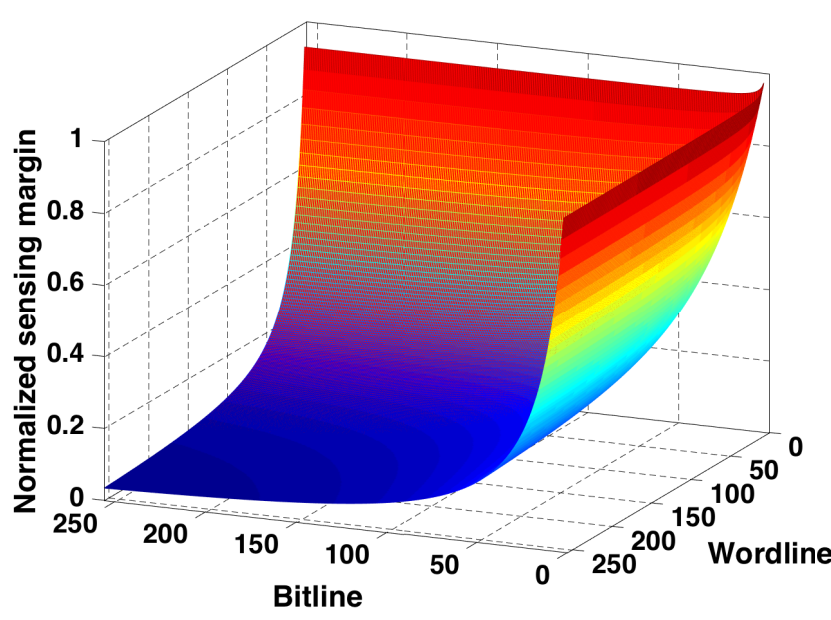

Fig. 8: Sensing margin degrades rapidly as array size increases in the presence of sneak path as computed using Eqn. 9. 


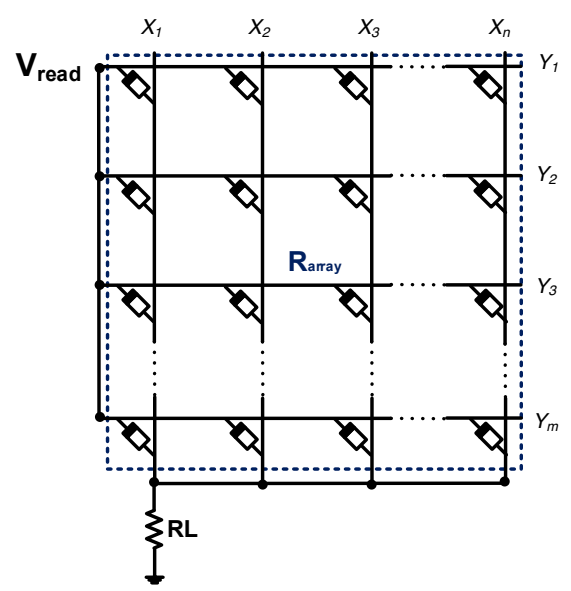

(a)

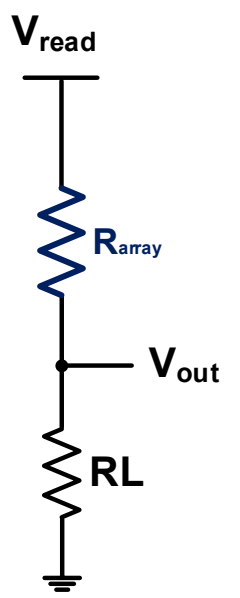

(b)
Fig. 9: (a) Sensing mechanism for a crossbar sensor made up of $m \times n$ sensors. (b) Equivalent circuit for sensing mechanism.

in a basic crossbar array cannot be used as a sensor on its own. We propose a structure that consists of an $m$ $\times \mathrm{n}$ crossbar array of memristors such that the en-tire array act as a single sensor. A parallel readout of the crossbar matrix could be achieved by connecting all the wordlines to the read voltage and all bitlines are grounded via the load resistor for sensing. The structure of the sensing mechanism and its equivalent circuit model is shown in Fig. 9a and 9b respectively. The response of this structure to any gas can be easily computed by measuring the overall resistance of memristor before and after interaction with the subject gas.

Measuring the response of a sensor that consist of an array of memristors will differ slightly from that of a single memristor shown in Fig. 6 . This is because the ratio of the initial and final resistance of the crossbar remains constant as the size varies with fixed gas con-centration. For simulation purpose, the overall output voltage of the array computed by Eqn. 12 will instead be used to measure the sensor's response.

$V_{\text {out }}=V_{\text {read }} \times \frac{R_{L}}{R_{L}+R_{\text {array }}}$

where $R_{\text {array }}$ is a parallel combination of all the memristance $\left(R_{\text {mem }}\right)$ in the array. Simulation result of this sensing technique shows that the sensor's response improves with increasing number of memristors in parallel as shown in Fig 10. Rate of improvement to the sensor's responsiveness is however not linear with increasing ar-ray size. The increment in response rate drops to approximately $1 \%$ once the number of devices approach $5 \mathrm{~K}$ and almost negligible by the time the 10, 000th de-vice is added to the array. Hence, an array size beyond $5 \mathrm{~K}$ is not justifiable.

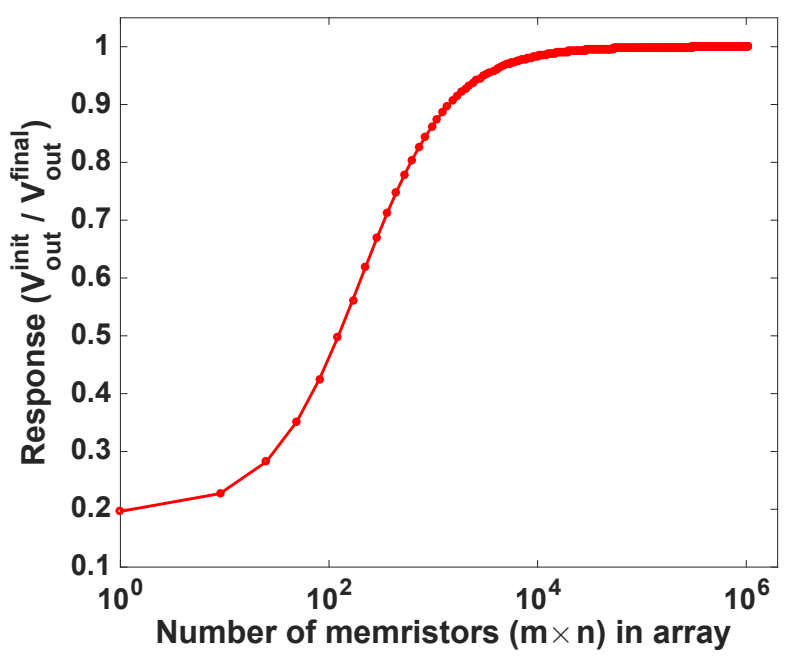

Fig. 10: Sensor's response increases as the number of memristors in the $\mathrm{m} \times \mathrm{n}$ sensor structure increases. $\mathrm{R}_{\text {on }}=$ $1 \mathrm{~K} \Omega, \mathrm{R}_{\mathrm{L}} \ll \mathrm{R}_{\text {on }}, \mathrm{C}=10^{4} \mathrm{ppm}$.

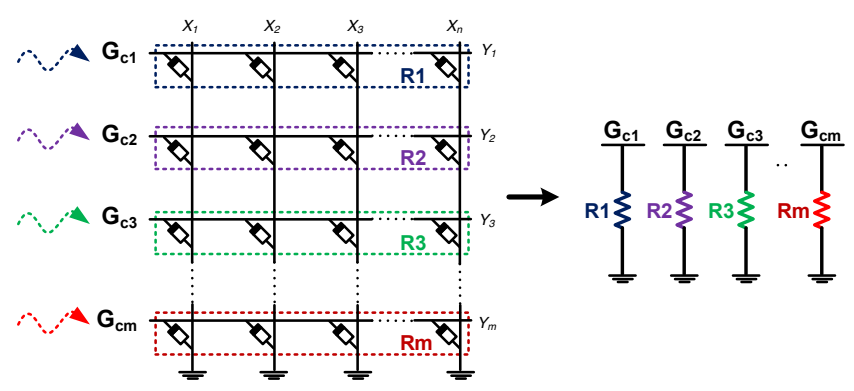

Fig. 11: The proposed multi-sensing structure. An $m \times n$ array of multiple sensors able to detect $m$ different gases and its equivalent circuit model

\subsection{Multi-Gas with $m(1 \times n)$ Sensor Array}

This section proposes a crossbar structure suitable for sensing multiple gases. A matrix of memristor sensors are designed such that the memristors in the same row have identical initial properties that enables them to react in approximately similar pattern in the presence of any target gas. Preliminary results from this sens-ing structure was presented in [46]. In this architecture, each row in the matrix acts as a sensor. The number of gases that can be sensed using this architecture de-pends on the number of rows in the crossbar as shown in Fig. 11. The number of sensing devices in each row can be determined based on the level of redundancies or samples needed in order to decide the properties of the target gas. All the memristors in each row will have the same properties and we assume negligible line resis-tance. The sneak-path effect can be eliminated in this 


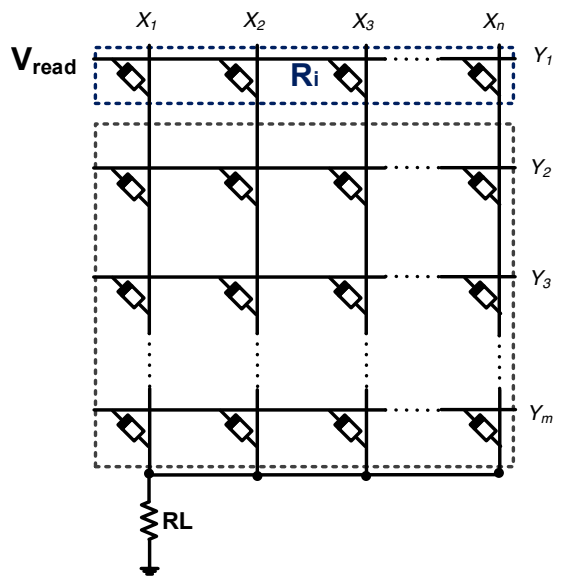

(a)

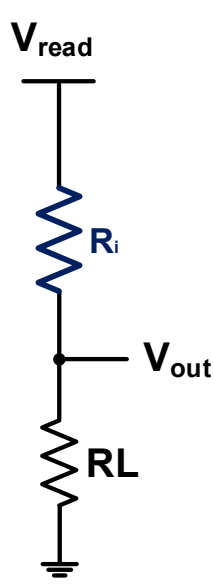

(b)
Fig. 12: (a) Reading technique for the multi-sensing structure (b) Corresponding equivalent circuit model.

structure by sensing all memristors in each of the rows simultaneously while the columns are shorted as shown in Fig. 12. Also, the presence of line resistance does not introduce sneak-paths current into the sensing circuit. For simulation purpose, the overall output voltage of any of the mth row sensor can be computed by Eqn. 13 will instead be used to measure the sensor's response.

$V_{\text {out }}=V_{\text {read }} \times \frac{R_{L}}{R_{L}+R_{i}}$

where $R_{i}$ is a parallel combination of all the memris-tance $\left(R_{\text {mem }}\right)$ in the target row $(1 \leq i \leq m)$. All the memristors in a row will have approximately similar resistance value because of their identical initial con-ditions. Also note that when the resistance value of each row is being sensed, all other memristors in the array are shunted out of the circuit and they do not contribute to the sensed output. $m$ number of reads is required to detect the response of all the sensors in the $\mathrm{m} \times \mathrm{n}$ crossbar. Fig. 13 shows the simulation re-sult from directing a range of reducing gas concentra-tion to a row of a $4 \times 4$ sensor array. The initial resis-tance value of the four memristors in the target row was set to $100 \Omega, 123 \Omega, 127 \Omega$ and $140 \Omega$, representing an ex-treme case of almost $33 \%$ variation between the smallest and largest memristance. Despite the large variation, an average response (red waveform) which represents the parallel combination of the four memristor was mea-sured by the sensing architecture over the range of gas concentration (pink waveform). If all the rows in the crossbar are used for gas sensing, measurements will be done by a succession of $m$ read steps for $i=1, \ldots, m$ and all responses recorded accordingly. The sensing struc-ture depicted by Fig. 11 also makes it possible to design a fault tolerant system by introducing a repair mech-anism that addresses faulty sensors in the array. This

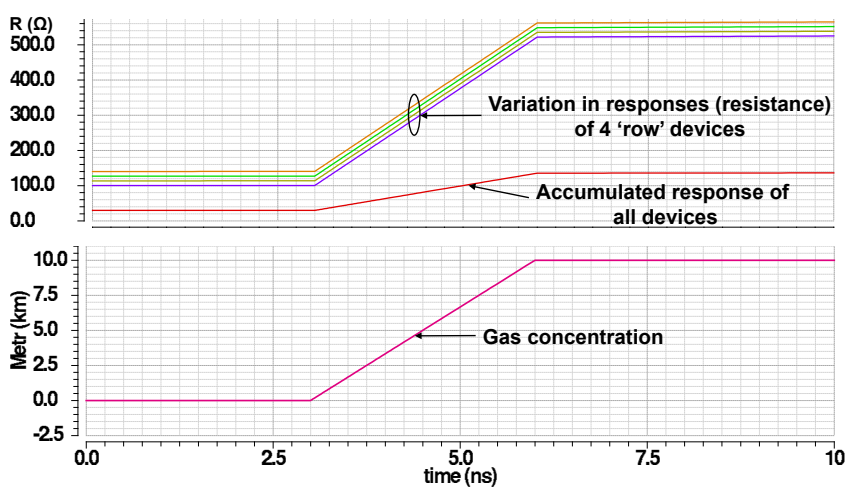

Fig. 13: Simulation result of exposing a row from a $4 \times 4$ memristor crossbar array to a range of gas concentration (reducing gas).

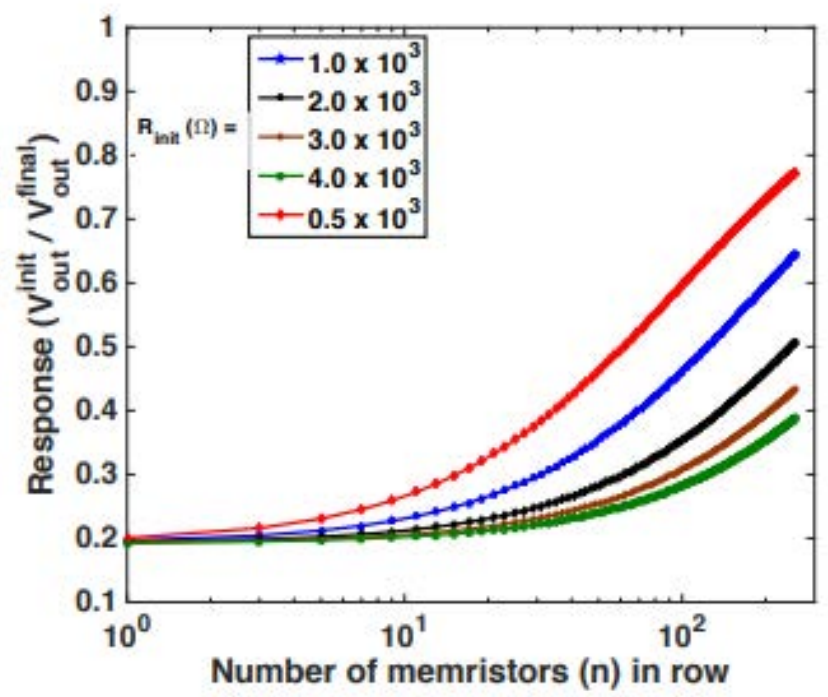

Fig. 14: Response of five rows of memristors to gas presence. All memristors in the same row are set to same initial resistance.

can be achieved by checking if the response of a memristor (sensor) considerably differs from the rest, in such case, the resistance of the cell can be set to a high resistance state or such cell substituted with a spare one. As clearly shown in Fig. 14, response of the row-based sensor gets better as the number of memristors in the row increases. In this simulation, a crossbar with five rows was created and each row initialised to different $R_{\text {on }}$ values. The number of memristors in each row was progressively increased and result shows improved re-sponse as the number of sensing memristor grows across the five rows in the crossbar. Measurements was done by a succession of $\mathrm{m}$ reads for $\mathrm{i}=1, \ldots, \mathrm{m}$ and each of the responses are recorded. 


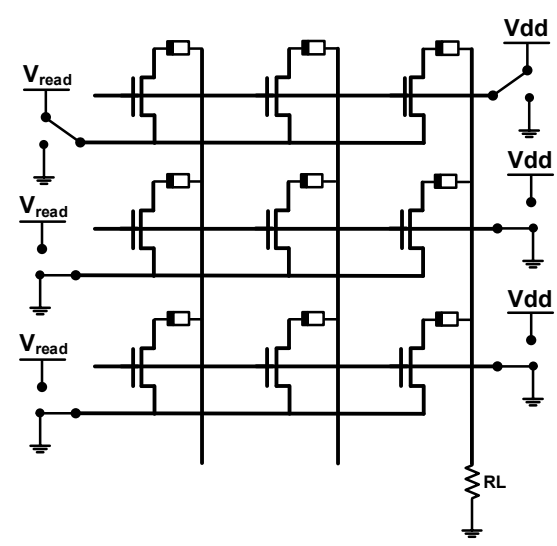

Fig. 15: One transistor, one memristor structure for minimising sneak-path effect in an array of sensors. The sensor in the top right corner is selected for sensing.

\subsection{The $1 \mathrm{~T} 1 \mathrm{M}$ Structure}

$1 \mathrm{~T} 1 \mathrm{M}$ is another memristor-based architecture that solves the sneak-path problem [47]. The 1T1M architecture shown in Fig. 15 consist of an access transistor and a memristor at every cross-point. The transistor enable independent access to each memristor cell without in-terference from other cells but at the expense of area per cell. The 1T1M structure has a bigger footprint because of the access transistor. To perform the sensing opera-tion in this architecture, only the row that contains the target cell is enabled via the access transistor and only the corresponding column grounded. In this way, the desired sensor can be read without sneak-path and each cell can be used to independently sense different gases. Response of each memristor in the array will be similar to the simulation result of an isolated single memristor in Fig. 6. Clearly the 1T1M approach, which resembles the DRAM architecture, does not leverage the poten-tial density improvement achievable in a purely memris-tive crossbar structure. This approach can be adopted whenever high density is less of a priority.

\section{Discussion of Simulation Results}

As earlier mentioned, the accuracy and relia-bility of gas sensors can be improved statisti-cally by taking multiple measurements from the same sensor or from different independent sen-sors. This section examines the accuracy of the three proposed sensing structures. An impor-tant figure of merit is the resistance swing of a gas sensor. We define resistance swing as the window between the maximum and minimum values in the resistance distribution of the sens-ing device after measurement. A reliable sen- sor will have a small resistance swing which will make it possible to detect slight changes in gas concentration. In order to estimate the resis-tance swing of the proposed sensing structures, we simulate multiple measurements with mul-tiple samples. The initial and final resistance values of $5 \times 10^{4}$ samples of each of the sens-ing structures described in Section 5 were mea-sured. We assume a 5\% standard deviation in the initial resistance value of each memristor used in the sensor, the same standard deviation was also applied to the final resistance after $10^{3} \mathrm{ppm}$ volume of gas was applied. The aim of applying the deviation to the resistance values is to evaluate how well each structure can toler-ate resistance variation that often affects mem-ristive devices. Fig. 16 shows the Monte Carlo simulation of resistance distribution using $5 \times 10^{4}$ samples from each of the sensing structures de-scribed in Section 5 . The $m \times n$ structure has the least resistance swing before and after gas exposure. The presence of more memristors in the $\mathrm{m} \times \mathrm{n}$ led to a desirable reduction in the standard deviation of the overall resistance of the array. The $m \times n$ structure benefits from the idea of averaging the response of multiple mem-ristors in order to increase the sensor's reliabil-ity and precision as shown in Fig. 16a. Similarly, the $\mathrm{m}(1 \times \mathrm{n})$ structure has a fairly less standard deviation (Fig. $16 b)$ compared with that of the single memristor in Fig. 16c. Simulation results presented in Fig. 16a, 16b and $16 \mathrm{c}$ were per-formed using a single memristor sensor cell, $1 \times 8$ and $8 \times 8$ sensor array respectively. A possible $5 \%$ standard deviation in memristance could invari-ably lead up to a $60 \%$ progressive variation in the sensor response over a sample size of $5 \times 10^{4}$ sin-gle memristors. However, the same sample size with a $8 \times 8$ sensor array reduces the variation in the sensor responses to as low as $5.3 \%$. This makes the $m \times n$ structure a more reliable option due to the averaging effect of having more mem-ristors in the sensing block. The $m \times n$ is however limited to sensing a single gas at a time unlike the $m(1 \times n)$ and $1 T 1 M$ structures that can sense multiple gases. The numbers of gases that can be sensed depends on the number of rows $(\mathrm{m})$ and number of memristors in the array respec-tively. The $m \times n$ structure gives the lowest re-sistance swing compared to the other two struc-tures because of less variation in its measured response. Table 2 shows the comparison of the different proposed structures. These sensing ap- 


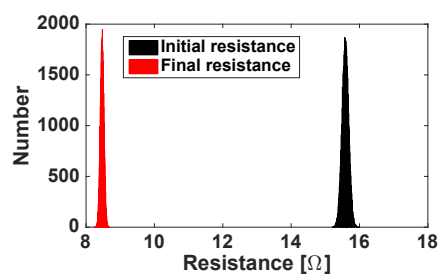

(a)

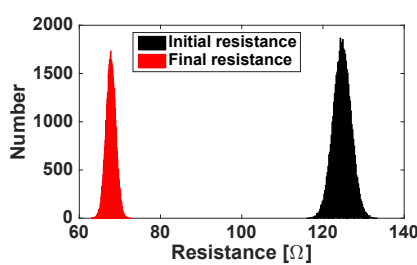

(b)

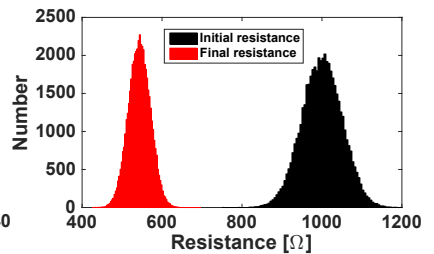

(c)
Fig. 16: Initial and final resistance distribution in (a) $m \times n$ sensing structure as explained in section 5.1 , (b) $1 \times n$ sensor representing one of the $m$ sensors used for multigas sensor explained in section 5.2 and (c) a single memristor sensor from use of the 1T1M structure. $m=8, n$ $=8, \mathrm{C}=10^{3} \mathrm{ppm}$.

Table 2: Relative comparison of sensing structures

\begin{tabular}{|l|c|c|c|}
\hline & $\boldsymbol{m} \times \boldsymbol{n}$ & $\mathbf{1} \times \boldsymbol{n}$ & $\mathbf{1 T 1 M}$ \\
\hline Reliability & High & Moderate & Low \\
\hline Sneak-path & No & No & No \\
\hline Resistance Swing & Low & Moderate & High \\
\hline Multi-Gas sensing & No & $\begin{array}{c}\text { Yes } \\
\text { (number of rows) }\end{array}$ & $\begin{array}{c}\text { Yes } \\
\text { (number of cells) }\end{array}$ \\
\hline
\end{tabular}

proaches are essential for integrating very dense sensor arrays on well known and established memory-like architectures already proposed for memristive arrays. As earlier implied, memristive sensors can be lumped together to function as a single sensor, unlike memristorbased memory array, where the content of individual device has to be explicitly measured to make sense of the data. For the purpose of gas detection, averaging responses from multiple sensing devices enhances the sensor's overall accuracy. This averaging technique is achieved via parallel combination of all concerned de-vices which helps to knock out the effect of any fault within the crossbar array. Another interesting aspect of memristor's use as sensor in crossbar architecture is the opportunity to sense multiple memristors without the sneak-path problem.

\section{Conclusions}

In this paper, we presented a comparative analysis of possible sensing memristor arrays architectures. The contribution of this work starts with the development of a gas sensitive Verilog-A memristor model. This model takes into account the already established gas sensing properties of $\mathrm{T} \mathrm{iO}_{2}$ memristors and could be expanded to other metal oxide materials. Using this model, three gas sensing techniques were proposed and investigated, namely, $m \times n$, $m(1 \times n)$ and 1 T1M. Our analysis shows $m \times n$ and $m(1 \times n)$ structures are more efficient in terms of responsiveness and reliability but at the expense of the number of gases that could be sensed. On the other hand 1T1M enables the deployment of high numbers of independent sensors in a single array. We believe that the proposed analysis represents a fundamental corner-stone to the success of this novel approach to sensing: future developments include the manufacturing, analy-sis, and modelling of prototypes based on the proposed architectures.

References

1. V. Bochenkov, G. Sergeev, Metal Oxide Nanostructures and Their Applications 3, 31 (2010)

2. N. Yamazoe, Sensors and Actuators B: Chemical 5(1), 7 (1991)

3. D.E. Williams, Sensors and Actuators B: Chemical 57(1), 1 (1999)

4. P. Clifford, D. Tuma, Sensors and Actuators 3, 233 (1982)

5. G.F. Fine, et al., Sensors 10(6), 5469 (2010)

6. A. DÁmico, C. Di Natale, IEEE Sensors Journal 1(3), 183 (2001)

7. T. Plecenik, M. Moško, A. Haidry, P.Ďurina, M. Truchỳ, B. Grančič, M. Gregor, T. Roch, L. Satrapinskyy, A. Mošková, et al., Sensors and Actuators B: Chemical 207, 351 (2015)

8. S. Vallejos, I. Grácia, O. Chmela, E. Figueras, J. Hubálek, C. Cané, Sensors and Actuators B: Chemical 235, 525 (2016) 9. D.B. Strukov, et al., nature 453(7191), 80 (2008)

10. A. Kay, M. Grätzel, Solar Energy Materials and Solar Cells 44(1), 99 (1996)

11. A. Fujishima, T.N. Rao, D.A. Tryk, Journal of Photo-chemistry and Photobiology C: Photochemistry Reviews 1(1), 1 (2000)

12. C. Garzella, E. Comini, E. Tempesti, C. Frigeri, G. Sberveglieri, Sensors and Actuators B: Chemical 68(1), 189 (2000)

13. Q. Wang, Y. Pan, S. Huang, S. Ren, P. Li, J. Li, Nanotechnology 22(2), 025501 (2011)

14. S. Carrara, et al., Sensors and Actuators B: Chemical 171, 449 (2012)

15. J. Fraden, Handbook of modern sensors: physics, designs, and applications (Springer Science \& Business Media, 2004)

16. L.O. Chua, Circuit Theory, IEEE Transactions on 18(5), 507 (1971)

17. J. Borghetti, G.S. Snider, P.J. Kuekes, J.J. Yang, D.R. Stewart, R.S. Williams, Nature 464(7290), 873 (2010)

18. X. Yang, A. Adeyemo, A. Jabir, J. Mathew, Electronics Letters 52(11), 906 (2016)

19. J. Cong, B. Xiao, in Nanoscale Architectures (NANOARCH), 2011 IEEE/ACM International Symposium on (IEEE, 2011), pp. 1-8 
20. A. Thomas, Journal of Physics D: Applied Physics 46(9), 093001 (2013)

21. F. Puppo, A. Dave, M.A. Doucey, D. Sacchetto, C. Baj-Rossi, Y. Leblebici, G. De Micheli, S. Carrara, NanoBio-science, IEEE Transactions on 13(1), 19 (2014)

22. J. Mathew, Y. Yang, M. Ottavi, T. Browna, A. Zam-petti, A. Di Carlo, A. Jabir, D.K. Pradhan, in Defect and Fault Tolerance in VLSI and Nanotechnology Sys-tems (DFTS), 2015 IEEE International Symposium on (IEEE, 2015), pp. 7-12

23. C.Y. Lin, D.Y. Lee, S.Y. Wang, C.C. Lin, T.Y. Tseng, Surface and Coatings Technology 203(5), 628 (2008)

24. H. Lv, M. Wang, H. Wan, Y. Song, W. Luo, P. Zhou, T. Tang, Y. Lin, R. Huang, S. Song, et al., Applied Physics Letters 94(21), 213502 (2009)

25. A. Mehonic, S. Cueff, M. Wojdak, S. Hudziak, O. Jam-bois, C. Labb é, B. Garrido, R. Rizk, A.J. Kenyon, Journal of Applied Physics 111(7), 074507 (2012)

26. S. Porro, E. Accornero, C.F. Pirri, C. Ricciardi, Carbon 85, 383 (2015)

27. H. Nili, S. Walia, S. Balendhran, D.B. Strukov, M. Bhaskaran, S. Sriram, Advanced Functional Materials 24(43), 6741 (2014)

28. H.S.P. Wong, H.Y. Lee, S. Yu, Y.S. Chen, Y. Wu, P.S. Chen, B. Lee, F.T. Chen, M.J. Tsai, Proceedings of the IEEE 100(6), 1951 (2012)

29. J.J. Yang, M.D. Pickett, X. Li, D.A. Ohlberg, D.R. Stewart, R.S. Williams, Nature nanotechnology 3(7), 429 (2008)

30. R. Williams, Spectrum, IEEE 45(12), 28 (2008)

31. A.A. Haidry, A. Ebach-Stahl, B. Saruhan, Sensors and Actuators B: Chemical (2017)

32. D. Williams, G. Henshaw, K. Pratt, R. Peat, Journal of the Chemical Society, Faraday Transactions 91(23), 4299 (1995)

33. Y. Xu, K. Yao, X. Zhou, Q. Cao, Sensors and Actuators B: Chemical 14(1), 492 (1993)

34. J. Bai, B. Zhou, Chemical reviews 114(19), 10131 (2014)

35. H.J. Kim, J.H. Lee, Sensors and Actuators B: Chemical 192, 607 (2014)

36. N. Barsan, C. Simion, T. Heine, S. Pokhrel, U. Weimar, Journal of Electroceramics 25(1), 11 (2010)

37. S. Naisbitt, et al., Sensors and Actuators B: Chemical 114(2), 969 (2006)

38. E. Linn, S. Menzel, R. Rosezin, U. Böttger, R. Bruch-haus, R. Waser, in 11th IEEE Conference on Nanotech-nology (IEEENANO) (IEEE, 2011), pp. 1474-1478

39. Y. Yang, J. Mathew, R.A. Shafik, D.K. Pradhan, Em-bedded Systems Letters, IEEE 6(1), 12 (2014)

40. Z. Biolek, D. Biolek, V. Biolkova, Radioengineering 18(2), 210 (2009)

41. R. Binions, et al., Journal of The Electrochemical Society 156(3), J46 (2009)

42. D. Batas, H. Fiedler, IEEE Transactions on Nanotech-nology 10(2), 250 (2011)

43. H. Sundgren, I. Lundström, F. Winquist, I. Lukkari, R. Carlsson, S. Wold, Sensors and Actuators B: Chemical 2(2), 115 (1990)

44. E.J. Wolfrum, R.M. Meglen, D. Peterson, J. Sluiter, Sen-sors and Actuators B: Chemical 115(1), 322 (2006)

45. E. Linn, R. Rosezin, C. Kügeler, R. Waser, Nature ma-terials 9(5), 403 (2010)

46. A. Adeyemo, A. Jabir, J. Mathew, E. Martinelli, C. Di Natale, M. Ottavi, in IEEE International Sym-posium on On-Line Testing and Robust System Design (IOLTS) (2017)
47. M. Zangeneh, A. Joshi, in Proceedings of the great lakes symposium on VLSI (ACM, 2012), pp. 9-14

48. X. Yang, A. Adeyemo, A. Bala, A. Jabir, in 27th International Symposium on Power and Timing Modeling, Optimization and Simulation (PATMOS, 2017), pp. 1-5

49. K. Sungho, K. Sae-Jin, K. Kyung Min, L. Seung Ryul, C. Man, C. Eunju, K. Young-Bae, K. Chang Jung, C. U-In, Y. In-Kyeong, Scientific reports 3(2013)

50. Sadi, Toufik, et al. IEEE International Confer-ence on Simulation of Semiconductor Processes and Devices (SISPAD, 2016), pp. 149-152 